Психология. Журнал Высшей школы экономики,

2011. T. 8, № 1. С. 139-149.

\title{
ВЛИЯНИЕ УРОВНЯ ГИПНАБЕЛЬНОСТИ НА ХАРАКТЕРИСТИКИ ФОНОВОЙ ЭЭГ
}

\author{
А.В. КИРЕНСКАЯ, В.Ю. НОВОТОЦКИЙ-ВЛАСОВ, \\ В.Е. СТЕПАНОВА, А.Н. ЧИСТЯКОВ, В.М. ЗВОНИКОВ
}

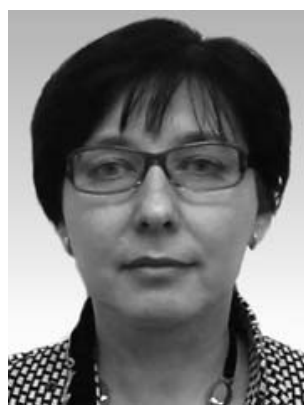

Киренская Анна Валерьевна - руководитель лаборатории ФГУ «ГНЦ социальной и судебной психиатрии им. В.П. Сербского Минздравсоцразвития», доктор биологических наук.

Сфера научных интересов - нейрофизиология, психофизиология, биологическая психиатрия, электроэнцефалография.

Контакты: neuro11@yandex.ru

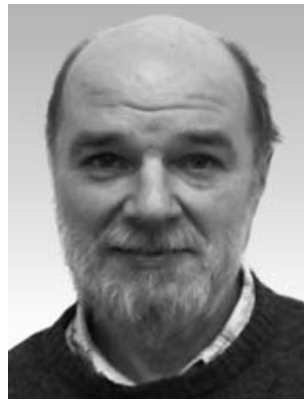

Новотоцкий-Власов Владимир Юрьевич - старший научный сотрудник ФГУ «ГНЦ социальной и судебной психиатрии им. В.П. Сербского Минздравсоцразвития», кандидат биологических наук.

Сфера научных интересов - нейрофизиология, электроэнцефалография, численные методы анализа.

Контакты: vnovot@mail.ru

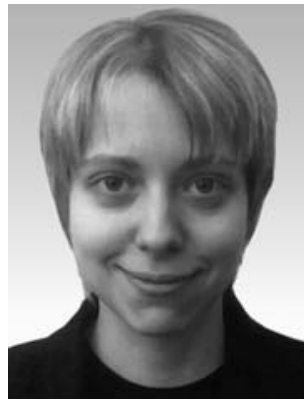

Степанова Валерия Евгеньевна - младший научный сотрудник ФГУ «ГНЦ социальной и судебной психиатрии им. В.П. Сербского Минздравсоцразвития».

Сфера научных интересов - психология, психофизиология.

Контакты: fioring@mail.ru 


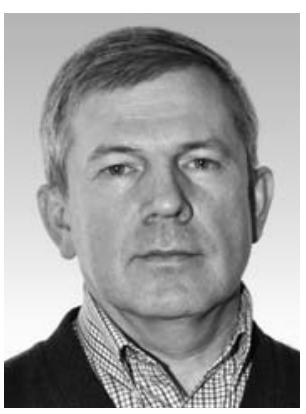

Чистяков Андрей Николаевич - научный сотрудник ФГУ «ГНЦ социальной и судебной психиатрии им. В.П. Сербского Минздравсоцразвития».

Сфера научных интересов - психология, психофизиология, методики психокоррекции.

Контакты: marketolog@autonet.ru

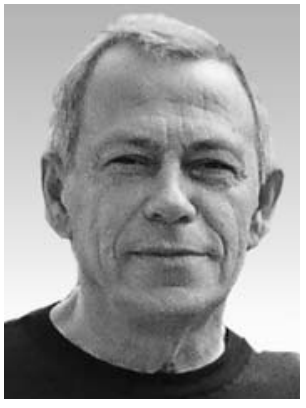

Звоников Вячеслав Михайлович - заместитель заведующего кафедры Московского гуманитарного университета, доктор медицинских наук, профессор.

Сфера научных интересов - психология, психофизиология, гипноз и гипнотерапия.

Контакты: vzvonikov@yandex.ru

\section{Резюме}

Исследования проведены с участием 30 добровольчев, из которых 18 человек были высокогипнабельными (ВГ) и 12 человек - низкогипнабельными (НГ).

Запись ЭЭГ (19 стандартных отведений) осуществляли в состоянии спокойного бодрствования с закрытыми глазами в течение 2 минут. Анализировали спектральную мощность (СМ) и когерентность (КОГ). Подтверждены данные других авторов о повышенной СМ тета-диапазона у ВГ лии. Впервые показано, что ВГ лича характеризуются значительно более высоким уровнем КОГ в тета- и альфа-диапазонах ЭЭГ по сравнению с НГ. СМ и КОГ высокочастотных диапазонов (бета2 и гамма) в группе ВГ, напротив, были ниже, чем в группе НГ. В целом полученные результаты позволяют предположить, что ВГ лицам более свойственно образное мышление, а НГвербально-логическое.

Ключевые слова: гипнабельность, спектр ЭЭГ, когерентность.

\section{Введение}

Уровень гипнабельности (Г) является устойчивой личностной характеристикой (Piccione et al., 1989; Kumar et al., 1996). Психологические и психофизиологические исследова- ния показали, что уровень гипнабельности положительно коррелирует с концентрацией внимания, яркостью внутренних образов, эмоциональностью, воображением, творческими способностями (Crawford, 1989; Crawford et al., 1993; Crawford et al., 
1995; Crowson et al., 1991; Gruzelier, 2002). Кроме того, определение уровня гипнабельности важно для психотерапии, так как он влияет на выбор тактики психотерапевтических мероприятий и их эффективность (Gruzelier, 1996, 2002; Kirenskaya et al., in press; Spiegel, 2007). Изучение биоэлектрической активности мозга у лиц с разным уровнем гипнабельности является актуальным для понимания ее центральных механизмов.

Проводившиеся ранее исследования позволили обнаружить взаимосвязь уровня гипнабельности с определенными частотными и региональными характеристиками ЭЭГ в состояниях бодрствования и гипнотического погружения (Crawford et al., 1996; De Pascalis, 1999; Sabourin et al., 1990; Williams, Gruzelier, 2001).

В большинстве работ обнаружена повышенная тета-активность у высокогипнабельных лиц, а также ее увеличение в состоянии гипноза (Graffin et al., 1995; Sabourin et al., 1990; Williams, Gruzelier, 2001).

Противоречивые результаты были получены для гамма-активности ЭЭГ в диапазоне около 40 Гц. В ранних исследованиях обнаружена исходно сниженная активность в этом диапазоне у высокогипнабельных испытуемых (ВГ) по сравнению с низкогипнабельными (НГ) (De Pascalis et al., 1989). Однако позднее были получены обратные результаты: в состояниях спокойного бодрствования как с закрытыми, так и с открытыми глазами активность в диапазоне 40 Гц была выше у ВГ, чем у НГ (De Pascalis, 1993, 1999). Еще в одной работе также была найдена повышенная спектральная мощность ЭЭГ в диапазоне 36-44 Гц для подгруппы вы- сокогипнабельных лиц, демонстрирующих постгипнотическую амнезию (Schnyer, Allen, 1995).

Работы по изучению внутрикорковой синхронизации активности мозга, которая наиболее часто оценивается по показателю когерентности, у лиц с разным уровнем гипнабельности практически отсутствуют. Вместе с тем особенности внутри- и межполушарного взаимодействия играют важную роль в обеспечении когнитивных и эмоциональных процессов, а их изучение является необходимым для более глубокого понимания нейронального субстрата, опосредующего формирование нормальных и патологических функциональных состояний мозга (Болдырева, 2000; Свидерская, 1987).

Таким образом, влияние уровня гипнабельности на биоэлектрическую активность мозга остается малоизученным. Это обусловлено в основном отсутствием исследований, выполненных на современном методическом уровне. Перечисленные работы были проведены с использованием малого количества отведений ЭЭГ (от 2 до 6 электродов). Разными были локализация и монтаж электродов, а также методы анализа ЭЭГ.

Задачей настоящего исследования явилось применение современных методов регистрации и анализа многоканальной ЭЭГ с целью изучения нейрофизиологических механизмов гипнабельности и выявления ее ЭЭГ-коррелятов.

\section{Материалы и методы}

В исследовании приняли участие 30 психически и неврологически 
здоровых добровольцев в возрасте от 19 до 52 лет (средний возраст $34.4 \pm$ 2.0 года). Все испытуемые дали письменное согласие на участие в исследовании.

Уровень гипнабельности (Г) определяли с помощью модифицированной версии Стенфордской шкалы гипнабельности для группы (SHSS:C; Crawford, Allen, 1982) по 6 позициям по 5 баллов каждая. 18 человек (12 женщин и 6 мужчин) вошли в группу высокогипнабельных лиц (группа ВГ, $\Gamma=4.2 \pm 1.2), 12$ человек (6 женщин и 6 мужчин) - в группу низкогипнабельных лиц (группа НГ, Г = $1.5 \pm$ $0.11)$. По уровню гипнабельности различия между группами были достоверными $(p<0.001)$.

Во время исследования испытуемые находились в звукоизолированной камере. Запись ЭЭГ осуществляли в состоянии спокойного бодрствования с закрытыми глазами в течение 2 минут. ЭЭГ регистрировали от 19 стандартных отведений (по системе $10-20)$ с постоянной времени 0.3 с и верхней границей частотного фильтра 70 Гц на нейрокартографе фирмы «МБН» (Россия). Частота квантования сигналов составляла 200 Гц. Референтом служили объединенные ушные электроды.

ЭЭГ подвергали традиционному спектральному анализу. Спектральную мощность и когерентность вычисляли для 10 частотных диапазонов: дельта (1-3.5 Гц), тета1 (3.5-6 Гц), тета2 (6-8 Гц), альфа1 (8-10 Гц), альфа2 (10-11.5 Гц), альфа3 (11.513 Гц), бета1 (14-19 Гц), бета2 (1927 Гц), гамма1 (27-40 Гц) и гамма2 (41-59 Гц).

Для количественной оценки спектра ЭЭГ в каждом частотном диапа- зоне использовали натуральный логарифм абсолютной спектральной мощности (CM) и когерентность (КОГ) между всеми отведениями (171 пара электродов). С тем чтобы подавить ложную составляющую КОГ, связанную с общими референтами, и избежать возможного влияния мышечного напряжения, вычисляли частную когерентность, вводя поправку на активность в референтных отведениях (Bendat, Piersol, 1986).

Статистический анализ зависимых переменных проводился по стандартной схеме с использованием пакета статистических программ SPSS 11.0.

CM анализировали с помощью дисперсионного анализа ANOVA по факторам: Группа (2 уровня), Область коры (8 уровней), Полушарие (2 уровня) и их взаимным сочетаниям. Достоверность различий средних значений оценивали с помощью $\mathrm{t}$-критерия Стьюдента.

В связи с тем, что распределения показателя КОГ не соответствуют нормальному, межгрупповые сравнения средних значений этого показателя проводили с помощью непараметрического критерия Манна-Уитни.

\section{Результаты}

Согласно полученным результатам, параметры ЭЭГ в группах высоко- и низкогипнабельных лиц существенно различались.

В группе ВГ СМ тета-диапазона была существенно выше, а бета2- и гамма1-диапазонов, напротив, ниже, чем в группе НГ (рисунок 1).

Статистический анализ показателя спектральной мощности вы- 
Натуральный логарифм спектральной мощности (по оси ординат) тета1-, тета2-, бета2- и гамма1-диапазонов в группах высоко- (ВГ) и низкогипнабельных (НГ) лиц. Звездочками указаны достоверные межгрупповые различия: * $-p<0.05$

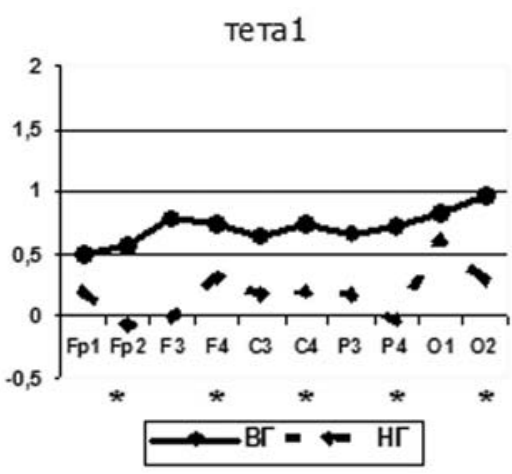

6 ета2

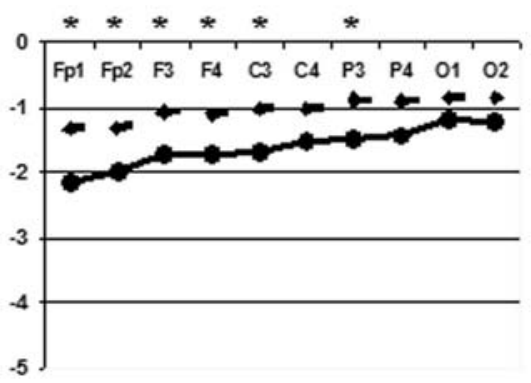

$\longrightarrow \mathrm{Br}=-\mathrm{Hr}$

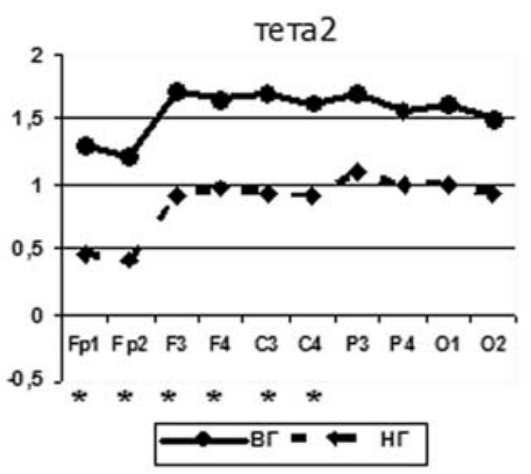

гамма 1

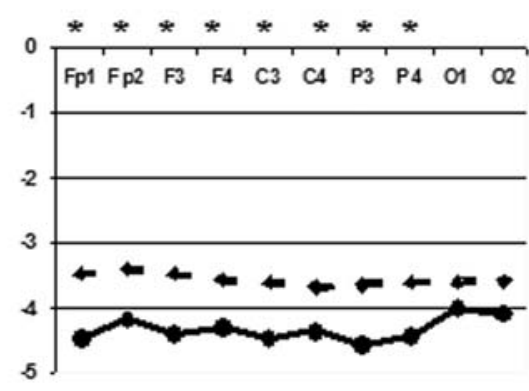

$\longrightarrow \mathrm{Br} \rightleftharpoons \mathrm{Hr}$

явил значимый эффект взаимодействия Группа $\times$ Область для СМ диапазона тета1 $(\mathrm{F}(7,196)=5.13$, $p<0.001)$. Для СМ диапазона тета2 найдены тенденция к значимости фактора Группа $(\mathrm{F}(1,28)=4.02, p=$ $=0.055)$ и значимый эффект взаимодействия Группа $\times$ Область $(\mathrm{F}(7$, $196)=2.60, p<0.05)$. Сравнение средних выявило в группе ВГ достоверно повышенную активность в тета1- и тета2-диапазонах в лобно-центрально-теменной области коры: в диапазоне тета1 - в отведениях Fp2, F4,

$\mathrm{Cz}, \mathrm{C} 4, \mathrm{Pz}, \mathrm{P} 4$ и $\mathrm{O} 2$, в диапазоне тета2 - в отведениях Fp1, Fp2, Fz, F3, $\mathrm{F} 4, \mathrm{~F} 7, \mathrm{~F} 8, \mathrm{Cz}, \mathrm{C} 3$ и $\mathrm{C} 4$ (рисунок 1). При проведении дополнительного анализа ANOVA по факторам Группа $(n=2)$, Область $(n=5: \mathrm{Fp} 1-2, \mathrm{~F} 3-4$, С3-4, P3-4, О1-2) и Полушарие $(n=2)$ фактор Группа был значимым как для тета1- $(\mathrm{F}(1,28)=5.04, p<0.05)$. так и для тета2-диапазонов ( $\mathrm{F}(1$, 28) $=4.73, p<0.05)$.

Значимые межгрупповые различия по показателю СМ также были получены для высокочастотных 
диапазонов бета2 и гамма1. В диапазоне бета 2 найдена тенденция к значимости фактора Группа $(\mathrm{F}(1,28)=$ $=3.61, p=0.068)$, а в диапазоне гамма1 фактор Группа был статистически значимым $(\mathrm{F}(1,28)=7.02$, $p<0.05)$.

Чтобы снизить возможное влияние мышечной активности, которая по частоте интерферирует с высокочастотными диапазонами ЭЭГ, особенно в маргинальных отведениях, дополнительный анализ был проведен по факторам Группа $(n=2)$, Область ( $n=3$ : F3-4, C3-4, Р3-4) и Латеральность ( $n=3$ : левые, медиальные и правые отведения). Значимые результаты также были получены для фактора Группа: в диапазоне бета2 $(\mathrm{F}(1,28)=3.95, p=0.057)$, в диапазоне гамма1 $(\mathrm{F}(1,28)=8.87$, $p<0.01)$. Анализ средних обнаружил достоверные различия $(p<0.05)$ в лобно-центрально-теменной области: в диапазоне бета2 - в отведениях Fp1, Fp2, F3, F4, F8, C3, P3, в диапазоне гамма1 - в отведениях Fp1,Fp2, Fz, F3, F4, Cz, C3, C4, Pz, P3, P4 (рисунок 1).

CM диапазона гамма2 также была выше в группе НГ по сравнению с группой ВГ, однако различия не достигали уровня значимости.

Еще более выраженные межгрупповые различия получены для показателя КОГ. В группе высокогипнабельных испытуемых уровень когерентности был значительно выше в дельта-, тета1-, тета2-, альфа1-, альфа2- и альфа3-диапазонах (рисунок 2). При этом в диапазоне тета1 достоверные различия найдены для 142 пар отведений, в диапазоне тета2 для 165 пар отведений, в диапазоне альфа3 - для 131 пары (рисунок 2B).
Следует также отметить, что величина КОГ, превышающая 0.5 , в группе ВГ выявлена для 16\% пар отведений в диапазоне тета1 и для 50\% пар в диапазоне альфа1, а в группе НГ для $0.5 \%$ и $29 \%$ пар отведений в тета1- и альфа1-диапазонах соответственно.

В диапазонах бета2 и гамма1 более высокие значения КОГ найдены для группы НГ, при этом статистически значимо КОГ была повышена в 22 парах отведений в диапазоне бета2 и в 74 парах - в диапазоне гамма1 (рисунок 2). Исключение составили показатели КОГ между передними и задними областями коры, которые были выше в группе ВГ. В диапазоне бета2 КОГ была выше в группе ВГ между следующими отведениями: с уровнем значимости $p<0.001-\mathrm{Fp} 1-\mathrm{O} 1, \mathrm{Fp} 1-\mathrm{O} 2, \mathrm{~F} 7-\mathrm{T} 6$ и с уровнем значимости $p<0.01-$ Fp1-P3, Fp1-O2, Fp1-T6, Fp2-O2, $\mathrm{Fp} 2-\mathrm{Pz}, \mathrm{F} 7-\mathrm{O} 2, \mathrm{~F} 8-\mathrm{T} 6$. В диапазоне гамма1 КОГ была выше в группе ВГ между отведениями Fp1-O1, Fp2-O2, Fp1-O2 с уровнем значимости $p<$ $<0.001$ и между отведениями Fp1-O2, F7-T6 с уровнем значимости $p<0.01$.

\section{Обсуждение}

Таким образом, проведенное исследование выявило набор спектральных и когерентных характеристик ЭЭГ, статистически значимо различающихся у испытуемых с высоким и низким уровнем гипнабельности.

Полученные данные, свидетельствующие о повышенной спектральной мощности в тета-диапазоне у высокогипнабельных лиц, согласуются с результатами ранее проведенных 


\section{Межгрупповые различия по показателю когерентности (КОГ)}

A

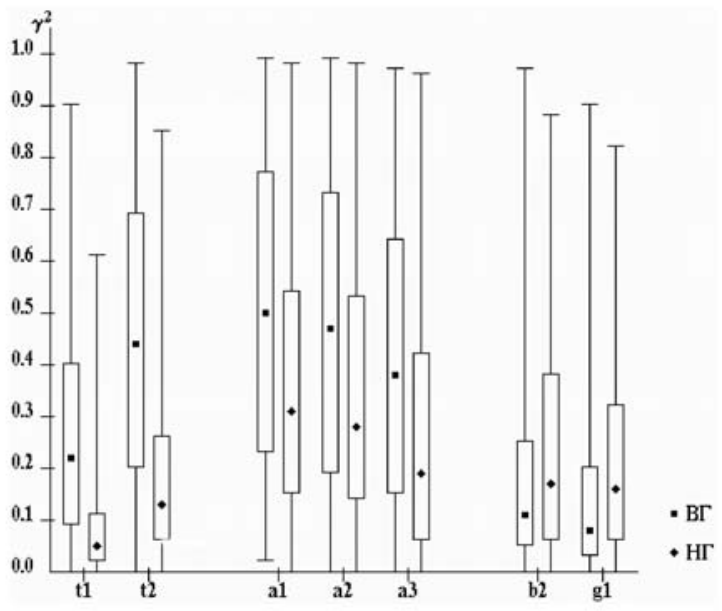

$\mathbf{D}$

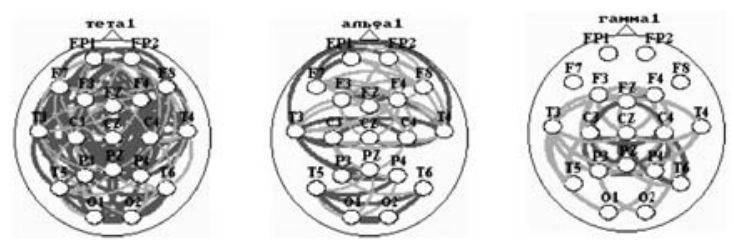

B

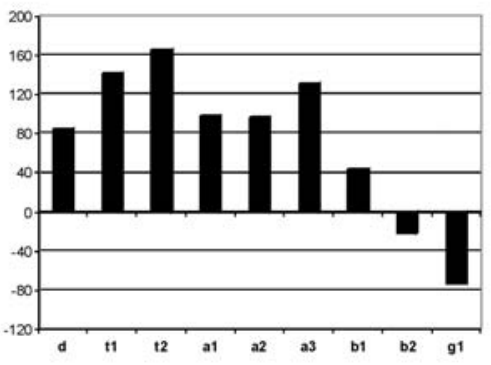

Примечания. Рисунок 2А. Значения КОГ (по оси ординат) в тета- (t), альфа- (a), бета- (b) и гамма- (g) диапазонах в группах ВГ и НГ лиц. - - медианы групп ВГ и НГ, $\quad-25-75 \%$, Iминимум - максимум. Рисунок 2Б. Карты достоверных межгрупповых различий по КОГ. Для тета1-, альфа1-диапазонов - значения КОГ выше в группе ВГ, для гамма1-диапазона - значения КОГ выше в группе НГ. $p<0.001-$ толстые темные линии, $p<0.01-$ тонкие светлые линии.

Рисунок 2B. Количество пар отведений, между которыми КОГ статистически значимо различается между группами в дельта- (d), тета- (t), альфа- (a), бета- (b) и гамма- (g) диапазонах. Положительные значения - КОГ выше в группе ВГ, отрицательные значения - КОГ ниже в группе ВГ. 
исследований (Sabourin et al., 1990; Graffin et al., 1995; Williams, Gruzelier, 2001). Значительное увеличение когерентности в диапазонах тета и альфа у высокогипнабельных лиц по сравнению с низкогипнабельными получено впервые.

При этом показано, что функциональные состояния, связанные с определенными когнитивными процессами, сопровождаются активационными изменениями показателей СМ и КОГ в одних и тех же частотных диапазонах.

Было показано, что низкие частоты ЭЭГ (от 1 до 10 Гц) имеют отношение к функциям восприятия, внимания, памяти (Klimesch, 1999; Weiss, Mueller, 2003; Sauseng, Klimesch, 2008). Усиление активности в тета-диапазоне связывают с такими процессами, как фокусированное внимание, воображение, выполнение зрительно-пространственных задач (Schacter, 1977; Rugg, Dickens, 1982; Klimesch, 1999). Повышенные показатели спектральной мощности и когерентности в частотной полосе тетаи альфа-диапазонов у высокогипнабельных испытуемых по сравнению с низкогипнабельными, в первую очередь, могут быть связаны с лимбической активацией и фасилитацией таламических синхронизирующих влияний на кору (Болдырева, 2000).

СМ и КОГ высокочастотных диапазонов бета2 и гамма1, напротив, были выше у лиц с низким уровнем гипнабельности. Активность этих диапазонов связана главным образом с когнитивными и лингвистическими функциями (Weiss, Mueller, 2003). Для гамма-диапазона обнаружена связь с широким спектром когнитивных процессов, включая внимание и репрезентацию образов (Lee et al., 2003). Интересно отметить, что в работе С. Вайс, Х. Мюллер (Weiss, Mueller, 2003) было показано, что генерация внутренних образов проявляется в увеличении когерентности в гамма-диапазоне между дистантными отведениями, расположенными в передних и задних областях коры. Согласно полученным нами результатам, в группе ВГ показатель КОГ был выше, чем в группе НГ, именно между фронтально-париетальными и фронтально-окципитальными парами отведений.

В целом полученные результаты позволяют заключить, что высокогипнабельным лицам более свойственно образное мышление, а низкогипнабельным - вербально-логическое. Косвенным подтверждением такого предположения является и высокий уровень дистантных внутрикорковых связей в тета-, альфа-, бета- и гамма-диапазонах, так как построение внутренних образов включает объединение разных сенсорных модальностей, связанных с разными отделами коры. Полученные результаты согласуются с выводами, сделанными в работе У. Рэя (Ray, 1997), в которой использовался нелинейный динамический анализ ЭЭГ.

Отдельного рассмотрения требуют сниженные показатели СМ и КОГ в гамма-диапазоне ЭЭГ, обнаруженные у высокогипнабельных лиц, которые некоторые исследователи рассматривают как генетический маркер шизофрении, или нейрофизиологический эндофенотип (van der Stelt, Belger, 2007). Ранее было найдено значимое снижение 
предстимульного торможения (ПСТ) стартл-реакции у высокогипнабельных испытуемых по сравнению с низкогипнабельными (Lichtenberg et al., 2007). По современным представлениям, дефицит ПСТ является одним из наиболее валидных кандидатов в эндофенотипы шизофрении (Braff, Freedman, 2002). Нейрофизиологические данные соотносятся с обширным фактическим материалом, полученным психологами и психиатрами о взаимосвязи между высокой гипнабельностью, шизотипическими личностными особенностями и склонностью к развитию психотических состояний (Gruzelier, 2002). Однако для понимания нейрофизиологических механизмов корреляции между исключительными когнитивными и творческими способностями высокогипнабельных лиц и повышенным риском к развитию психической патологии необходимы дальнейшие исследования.

\section{Заключение}

Таким образом, проведенное исследование обнаружило статистически достоверные различия спектральных и когерентных характерис-

\section{Литература}

Болдьрева Г.Н. Электрическая активность мозга человека при поражении диэнцефальных и лимбических структур. М.: Наука; МАИК «Наука/Интерпериодика», 2000.

Свидерская H.E. Синхронная электрическая активность мозга и психические процессы. М.: Наука, 1987. тик фоновой ЭЭГ у лиц с высоким и низким уровнем гипнабельности. Подтверждены данные других авторов о повышенной спектральной мощности тета-диапазона у высокогипнабельных лиц. Впервые показано, что высокогипнабельные испытуемые характеризуются значительно более высоким уровнем внутрикорковой синхронизации в тета- и альфа-диапазонах ЭЭГ по сравнению с низкогипнабельными. СМ и КОГ бета2- и гамма-диапазонов в группе ВГ, напротив, были ниже, чем в группе НГ. Исключение составили значения КОГ между лобными и теменно-затылочными областями коры в бета2- и гамма-диапазонах, которые были выше у высокогипнабельных лиц. В целом полученные результаты позволяют предположить, что высокогипнабельным лицам более свойственно образное мышление, а низкогипнабельным вербально-логическое.
Bendat J.S., Piersol A.G. Random data analysis and measurement procedures. N.Y.: Wiley, 1986.

Braff D.L., Freedman R. Endophenotypes in studies of the genetics of schizophrenia // K.L. Davis, D.S. Charney, J.T. Coyle, C. Nemeroff (eds.). Neuropsychopharmacology: The fifth generation of progress. 
Philadelphia: Lippincott Williams \& Wilkins, 2002. P. 703-716.

Craweford $H_{\text {: }}$. Cognitive and physiological flexibility: Multiple pathways to hypnotic responsiveness // V. Gheorghiu, P. Netter., H. Eysenck, R. Rosenthal (eds.). Suggestion and Suggestibility: Theory and research. N.Y.: Plenum Press; 1989. P. 155-168.

Crawford H.J., Brown A.M., Moon C.E. Sustained attentional and disattentional abilities: Differences between low and highly hypnotizable persons // J. Abnorm. Psychol. 1993. 102. 534-543.

Crawford H.J., Clarke S.W., Kitner-Triolo M. Self-generated happy and sad emotions in low and highly hypnotizable persons during waking and hypnosis: laterality and regional EEG activity differences // Int. J. Psychophysiol. 1996. 24. 239-266.

Craweford H.J., Kapelis L., Harrison D.W. Visual field asymmetry in facial affect perception: Moderating effects of hypnosis, hypnotic susceptibility level, absorption, and sustained attentional abilities // Int. J. Neurosci. 1995. 82. 11-23.

Croweson JJr., Conroy A.M., Chester T.D. Hypnotizability as related to visually induced affective reactivity // Int. J. Clin. Exp. Hypn. 1991. 39. 140-144.

De Pascalis V. EEG spectral analysis during hypnotic induction, hypnotic dream and age regression // Int. J. Psychophysiol. 1993. 15. 153-166.

De Pascalis V. Psychophysiological correlates of hypnosis and hypnotic susceptibility // Int. J. Clin. Exp. Hypn. 1999. 47. 117-143.

De Pascalis V., Marucci F.S., Penna P.M. 40-Hz EEG asymmetry during recall of emotional events in waking and hypnosis: differences between low and high hypnotizables // Int. J. Psychophysiol. 1989. 7. 85-96.

Graffin N.F., Ray W.J., Lundy R. EEG concomitants of hypnosis and hypnotic sus- ceptibility // J. Abnorm. Psychol. 1995. 104. 123-131.

Gruzelier J. The state of hypnosis: evidence and applications // Q. J. Med. 1996. 89. 313-317.

Gruzelier J. New insights into the nature of hypnotizability $/ / 2^{\circ}$ Simposio da Fundacao BIAL. Portugal, Porto, 2002. P. 275-293.

Kirenskaya A.V., Novototsky-Vlasov V.Y., Chistyakov A.N., Zvonikov V.M. The relations between hypnotizability, internal imagery and efficiency of NLP techniques // Int. J. Clin. Exp. Hypn., in press.

Klimesch W. EEG alpha and theta oscillations reflect cognitive and memory performanse: a review and analysis // Brain Research Review. 1999. 29. 169-195.

Kumar V.K., Pekala R.J., Cummings J. Trait factors, state effects, and hypnotizability // Int. J. Clin. Exp. Hypn. 1996. 44. 232-249.

Lee K.-H., Williams L., Breakspear M., Gordon E. Synchronous Gamma activity: a review and contribution to an integrative neuroscience model of schizophrenia // Brain Research Reviews. 2003. 41. 57-78.

Lichtenberg P., Even-Or E., Bar G., Levin R., Brin A., Heresco-Levy U. Reduced prepulse inhibition is associated with increased hypnotizability // Int. J. Neuropsychopharmacol. 2007. 11. 541-545.

Piccione C., Hilgard E., Zimbardo P. On the degree of stability of measured hypnotizability over a 25-year period // J. Pers. Soc. Psychol. 1989. 56. 289-295.

Ray W.I. EEG concomitants of hypnotic susceptibility // Int. J. Clin. Exp. Hypn. 1997. 3. 301-313.

Rugg M.D., Dickens A.M. Dissociation of alpha and theta activity as a function of verbal and visuospatial tasks // EEG and Clin. Neurophysiol. 1982. 53. 201-207.

Sabourin M.E., Cutcomb D.E., Crawford H. J., Pribram K. EEG correlates of hypnotic susceptibility and hypnotic trance: 
spectral analysis and coherence // Int. J. Psychophysiol. 1990. 10. 125-142.

Sauseng P., Klimesch W. What does phase information of oscillatory brain activity tell us about cognitive processes? //

Neurosci. Biobehav. Rev. 2008. 32. 1001-1013.

Schacter D.L. EEG theta waves and psychological phenomena: a review and analysis // Biol. Psychol. 1977. 5. 47-82.

Schnyer D.M., Allen J.J. Attention-related electroencephalographic and eventrelated potential predictors of responsiveness to suggested posthypnotic amnesia // Int. J. Psychophysiol. 1995. 43. 295-315.
Spiegel $H$. The neural trance: a new look at hypnosis // Int. J. Clin. Exp. Hypn. 2007. 55. 387-410.

Van der Stelt O., Belger A. Application of electroencephalography to the study of cognitive and brain functions in schizophrenia // Schizophr. Bull. 2007. 33. 4. 955-970.

Weiss S., Mueller H.M. The contribution of EEG coherence to the investigation of language // Brain Lang. 2003. 85. 325-343.

Williams J.D., Gruzelier J.H. Differentiation of hypnosis and relaxation by analysis of narrow band theta and alpha frequencies // Int. J. Clin. Exp. Hypn. 2001. 49. 185-206. 